\title{
Efficient Multi-Channel Signal Strength Based Localization via Matrix Completion and Bayesian Sparse Learning
}

\author{
Sofia Nikitaki, Grigorios Tsagkatakis, and Panagiotis Tsakalides
}

\begin{abstract}
Fingerprint-based location sensing technologies play an increasingly important role in pervasive computing applications due to their accuracy and minimal hardware requirements. However, typical fingerprint-based schemes implicitly assume that communication occurs over the same channel (frequency) during the training and the runtime phases. When this assumption is violated, the mismatches between training and runtime fingerprints can significantly deteriorate the localization performance. Additionally, the exhaustive calibration procedure required during training limits the scalability of this class of methods. In this work, we propose a novel, scalable, multi-channel fingerprint-based indoor localization system that employs modern mathematical concepts based on the Sparse Representations and Matrix Completion theories. The contribution of our work is threefold. First, we investigate the impact of channel changes on the fingerprint characteristics and the effects of channel mismatch on state-of-the-art localization schemes. Second, we propose a novel fingerprint collection technique that significantly reduces the calibration time, by formulating the map construction as an instance of the Matrix Completion problem. Third, we propose the use of sparse Bayesian learning to achieve accurate location estimation. Experimental evaluation on real data highlights the superior performance of the proposed framework in terms of reconstruction error and localization accuracy.
\end{abstract}

Index Terms_-Indoor localization, matrix completion, sparse Bayesian learning, multi-channel, received signal strength

\section{INTRODUCTION}

$\mathrm{R}$ ECENT technological developments in the sensing capabilities of smart phones have redefined the notion of mobile computing. Pervasive and ubiquitous computing requires location and mobility management in order to provide location dependent context. Potential applications of location aware mobile computing include health care monitoring, emergency management, personal tracking, and context dependent information services [1].

A typical localization scenario involves a set of Base Stations (BSs) placed at known positions and a Mobile Station (MS) that needs to be located. The BSs can be network enabled devices such as wireless mesh routers, wireless Access Points (AP), and sensor nodes, while the MS is a mobile device such as a smartphone. The actual localization can be performed either at a central station or at the mobile device. Certain localization schemes utilize the information from the BSs and require the MS to compute its own position, while other schemes utilize information from the MS to compute the location of the user remotely at the Localization Server (LS) [2]. In this work, we focus on the latter case since performing the localization at the LS offers

- S. Nikitaki is with the Network Research Division, NEC Laboratories Europe, Heidelberg, Germany. E-mail: Sofia.Nikitaki@neclab.eu.

- G. Tsagkatakis is with the Institute of Computer Science-FO.R.T.H, Crete, Greece.E-mail: greg@ics.forth.gr.

- P. Tsakalides is is with the Department of Computer Science, University of Crete, Greece, and the Institute of Computer Science-FO.R.T.H., Crete, Greece.E-mail: tsakalid@ics.forth.gr.

Manuscript received 1 June 2013; revised 30 Oct. 2014; accepted 12 Jan. 2015. Date of publication 18 Jan. 2015; date of current version 29 Sept. 2015.

For information on obtaining reprints of this article, please send e-mail to: reprints@ieee.org, and reference the Digital Object Identifier below.

Digital Object Identifier no. 10.1109/TMC.2015.2393864 certain benefits including lower power consumption at the mobile device and the ability to perform computationally demanding high level reasoning. This is important since, in spite of improvements in energy consumption, battery capacity grows slowly and power management is still a challenge in mobile computing. Moreover, remote location estimation facilitates the installation, maintenance, and management of the service.

A critical issue regarding the design of localization schemes is the type and characteristics of the measurements that are going to be used in the task. Due to the availability of different sensing modalities, numerous sensing platforms have been investigated for obtaining localization relevant data. Such modalities include laser rangefinders, ultrasounds, depth cameras, and Wi-Fi infrastructure signals. Of course, each modality is associated with a different set of characteristics including cost, data availability, reliability, form factor etc.

Selecting the appropriate data source, given the various constraints, is a subject under intense debate. For example in [3], the authors investigated different sensing modalities for robot localization including laser rangefinders, depth cameras, and Wi-Fi Received Signal Strength (RSS). Their analysis on real data indicated that although laser rangefinding can achieve the smallest absolute error, Wi-Fi signal strength achieves the lowest average localization error.

In this work, we consider Wi-Fi signals due to their abundance, reliability and acquisition ease. The key idea underlying Wi-Fi based localization schemes is that the power, and therefore the RSS, although it is affected by various factors in the propagation medium, is primarily dominated by the distance between the transmitter and the 
receiver. However, the layout and dynamics of physical spaces make the localization problem extremely demanding since signal power attenuation is heavily dependent on various phenomena including multipath, fading, shadowing, and the massive presence of objects (e.g., walls, obstacles, moving objects etc.),

Triangulation and scene analysis are the two principal techniques for location sensing. Triangulation or distance-based methods estimate the position of the mobile user by computing its distance from at least three reference points [4], [5], [6], [7]. The RSS measurements are translated to distance by applying theoretical or empirical path-loss models. However, formulating a reliable path-loss model is challenging and can lead to large localization errors [2].

To address this difficulty, scene analysis or fingerprinting techniques build training or signature maps to represent the physical space by capturing the variations of the indoor $\mathrm{Wi}$ Fi signal propagation [8]. To build a training map, an extensive calibration process is mandatory, where RSS fingerprints are collected at various locations [9], [10]. Although fingerprint-based systems can achieve high performance in terms of localization, the time and effort required during the training phase remain their major disadvantage.

From a network perspective, IEEE 802.11 is the dominant and most pervasive local wireless networking standard, making its extensive deployment and availability of infrastructure appealing also for positioning purposes. The radio frequency band of IEEE $802.11 \mathrm{~b} / \mathrm{g}$ is in $2.4 \mathrm{GHz}$, and is divided into 13 overlapping channels spaced $20 \mathrm{MHz}$ apart. Traditional IEEE 802.11 WLAN utilize a fixed-width channel allocation strategy where each AP uses a single channel for communication. Despite its low complexity, the single channel approach exhibits significant limitations, such as resource under-utilization, since it does not consider dynamically changing traffic conditions and requirements.

As a result, in recent years, Wi-Fi AP designers are keen to establish Dynamic Channel Assignment (DCA) strategies in order to boost network capacity and to avoid wasting RF resources [11]. Dynamic channel allocation strategies consider the temporary stochastic variations in traffic demand to provide optimal network coverage [11], [12]. Consequently, the associated channel per AP changes over time in order to successfully manage various network parameters such as mobility, user population, and demand of service, while minimizing the interference degradation between adjacent APs.

In addition to the IEEE 802.11 standard based communications, novel wireless communications architectures employ spectrum sharing among users and services to achieve various goals. For example, Cognitive Radio technology adapts the network operating parameters according to the surrounding radio environment resulting in stochastic DCA methods that could strongly affect RSS fingerprints [13]. Furthermore, multi-channel based localization is important for infrastructures which adopt Ultra-Wideband technologies. UWB technology utilizes a large frequency spectrum which could cause biases in the signal propagation estimates and ultimately lead to significant localization errors [14].

From a localization point of view, dynamic channel allocation has a direct impact on the RSS measurements since power attenuation across channels can vary in orders of

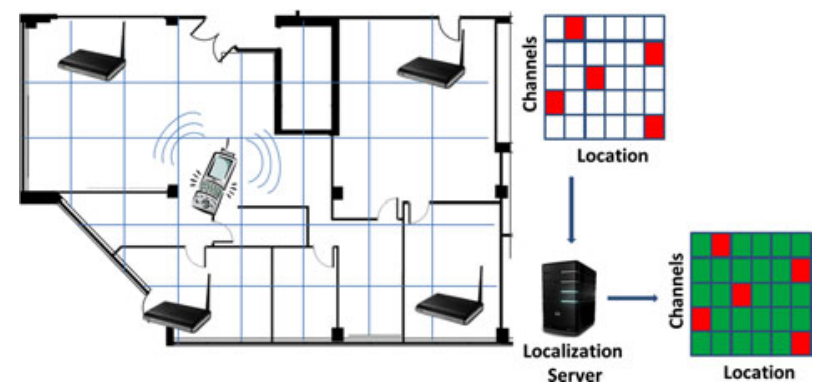

Fig. 1. A visual illustration of the fingerprint collection process during the training phase. Each Access Point records packets transmitted from the mobile device from multiple locations and at different channels. The incomplete signature maps are sent to the Localization Server which performs the MC based reconstruction to complete the missing entries. Note that the incomplete matrix shown in the figure corresponds to a single AP.

$\mathrm{dBs}$, suggesting large deviations between the RSS measurements at the same location but on different channels. Due to the existence of multiple communication channels, in a real world scenario it is possible that measurements collected during the runtime phase are compared with training measurements obtained from a different channel. Furthermore, for accurate positioning, a recalibration procedure is mandatory every time that environmental changes occur in the area of interest. Moreover training in multi-channel environments will be even more expensive and time consuming, since the signature map will consist of representative fingerprints at each possible spatial position and for every operational frequency.

In this work, we perform an in-depth study of the effects that multiple channels have on the RSS data collection process and we propose a novel multi-channel RSS fingerprintbased localization algorithm that exploits the signal power attenuation characteristics across space and frequency in order to provide accurate estimation while reducing the amount of training effort.

The rest of this paper is organized as follows: Section 3 presents an overview of related work concerning fingerprintbased systems. Section 4 discusses the motivation of the proposed framework. Section 4.3 presents the necessary MC background, while Section 5 describes the proposed multichannel localization approach. Experimental results are provided in Section 6, and the paper is concluded in Section 7.

\section{Overview of the Proposed Scheme}

Our proposed multi-channel localization scheme consists of two phases: the training phase and the runtime phase. We propose a reduced effort training phase where random channel sub-sampling is performed in order to collect RSS measurements on a grid of reference points from a subset of the available channels. The LS takes the partial signature maps and applies our proposed Matrix Completion (MC)based recovery technique in order to estimate the unobserved measurements and to build the complete channel-set signature map. A visual illustration of the training process is shown in Fig. 1.

During the runtime phase, the APs collect RSS measurements from the MS operating at a specific frequency, and location estimation is performed using the part of the 


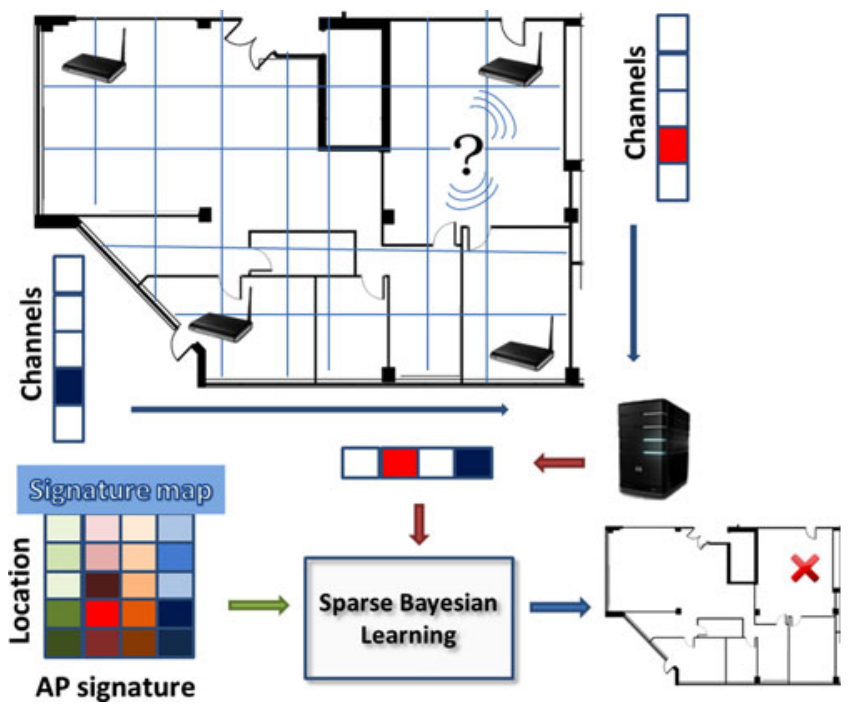

Fig. 2. A visual illustration of the localization process. Only the second (top right) and the fourth (bottom left) APs are in the same channel as the MS and collect runtime RSS measurements. The runtime measurements and the dictionary corresponding to the active channel are utilized by the Sparse Bayesian Learning (SBL) algorithm to identify the unknown location of the MS.

signature map associated to that frequency. A major contribution of this work is that we introduce for the first time a frequency-aware fingerprint-based localization system. Although the selected channel during runtime may be different from the one used during training, our MC approach provides the appropriate matching RSS measurements for localization. Furthermore, we propose a sparse Bayesian learning technique where localization estimates are obtained by searching for the sparsest solution of an underdetermined system of equations. An illustrative example of the runtime process is shown in Fig. 2.

In short, the main contributions of our system are:

- We explore the effect of multiple wireless frequency channels on RSS based fingerprint localization. This marks a significant departure from typical localization schemes that do not consider channel mismatch between the training and the runtime phases.

- We propose a novel training scheme, based on Matrix Completion, for efficiently generating a complete (across space and frequency) training signature map by randomly sub-sampling the available channels at various locations. The proposed training scheme substantially reduces the effort and time spent during training.

- Motivated by the inherent sparsity of indoor localization, we propose a novel location sensing technique based on Sparse Bayesian Learning. SBL is able to robustly identify the MS location by solving a under-determined system of equations arising from the recovered measurements.

- We experimentally validate our claims on real data collected from a representative active indoor office area that includes walls, cubicles, windows, and moving people. These experimental data allow the quantification of the effects of multiple channels on both state-of-art and the proposed technique.

\section{Related Work}

The plurality of localization approaches makes an extensive description of all the different paradigms very difficult. We therefore only consider fingerprint based localization approaches. Fingerprint based localization typically involves two phases: an offline/training phase where RSS fingerprints are collected at different locations in the target area and an online/runtime phase where received RSS measurements are compared with the training fingerprints to perform localization. In the following, we briefly describe current state of the art methods adopted by fingerprint based localization schemes.

\subsection{Training Phase: Calibration Techniques}

The construction of the signature map requires the collection of RSS fingerprints at each position within the area of interest. Fingerprint-based systems must allocate considerable resources, effort, and time in order to construct the signature map, even more so in dynamically changing environments where recalibration is mandatory [15]. As a consequence, researchers have striven to develop training techniques that attempt to shorten or even eliminate the training effort. Even in calibration-free systems, the benefit of the proposed method lies in the fact that it considers and incorporates the multichannel information.

With respect to minimizing calibration authors in [16] propose a training procedure where the whole area is divided into rooms, thereby limiting the possible locations to room-level granularity. Other localization protocols adopt data interpolation methods like kNN [17] and linear regression [18] to complete the training map using fingerprints taken at a small number locations. Recently, a Compressed Sensing (CS)-based training technique was proposed [19] that exploits the sparse nature of the RSS readings in the frequency domain to estimate the radio map from a small number of random measurements. Although this approach may reduce the number of locations used for training, reconstruction is performed independently for each AP and consequently the correlation among various APs is not taken into account leading to poor results.

To reduce the maintenance effort of the training phase, in [20] the signature map is estimated dynamically based on the runtime observations. To achieve this goal, neighbouring APs exchange RSS measurements during the runtime phase in order to update the signature map via a Gaussian Process regression model. Similarly, our proposed system is based on the measurements received at the APs. However, the calibration effort is reduced via channel sub-sampling and the inherent correlation structure of the sampled RSS fingerprints is considered for recovering the unobserved measurements.

To balance the performance degradation associated with a shortened calibration phase, the authors in [21] propose a hybrid generative/discriminative learning method that requires a small number of labeled samples and utilizes unlabeled samples to recover additional information for the target region area. Compared with the work in [21], our proposed Matrix Completion based training technique reduces the number of labeled data without requiring unlabeled data. 
Recently, a combination of various techniques has been proposed in order to minimize calibration effort. For example, Zee [22] incorporates inertial sensor measurements crowdsoursed from mobile users and Wi-Fi measurements sensed at predefined grids in order to combine dead-reckoning and RSS signature-based location sensing. However, the effectiveness of Zee could be hindered by the noisy built-in sensors of the mobile device and the number of crowdsourced measurements.

In order to reduce human effort during the training phase, one could employ robotic platforms to collect training data. However, since the training measurements must be associated with specific spatial locations, robotic platforms must also perform accurate Simultaneous Localization And Mapping (SLAM) using additional hardware such as lidar, cameras, ultrasound sensors, and laser scanners [3]. The two most important issues related to SLAM is the additional cost of the hardware and the location estimation errors that may render the training data useless. Even in this case however, collecting training data in a multi-channel environment via a traditional approach requires full frequency sweeping in order to construct representative signature maps. Such data may be unavailable, due to static network conditions during training, or hard to get (e.g., due to time/power constraints). As a consequence, the proposed channel sub-sampling approach could be also applied in scenarios where robotic platforms perform the training in order to substantially reduce the power consumption and time spent during calibration.

\subsection{Runtime Phase: Localization Techniques}

The majority of localization techniques fall under three categories with respect to the runtime phase: deterministic, probabilistic, and spatial sparsity-based.

Radar [9] is a deterministic indoor positioning system which combines signal strength measurements with specific signal propagation models to provide accurate location estimates. Position estimation is performed by the $k$-Nearest Neighbour in Signal Space algorithm (NNSS) [9], [17]. Another popular localization scheme is the Compass system [23] which utilizes the IEEE 802.11 infrastructure and digital compasses under a probabilistic framework to achieve low cost localization services.

Horus [24] is a map-based system, which considers different causes for the wireless channel variations. Horus employs a stochastic description of the signature map and performs localization via a maximum likelihood based approach. Within the probabilistic framework, Bayesian classification has been naturally adopted to address the localization problem [25], [26]. Authors in [27] proposed the mapping of the received measurements onto principal components (PCs) and adopt a probabilistic approach based on PCs in order to perform maximum likelihood localization.

Spatial sparsity based approaches exploit the sparsity of the position for localization, i.e., the fact that a MS can only be in one location at each given time. In [28], we reformulated the localization problem as a sparse approximation problem based on the Compressed Sensing theory that provides a new paradigm for recovering sparse signals by solving an $l_{1}$ minimization problem [29], [30]. In a similar vein, in [31] we considered a centralized localization protocol based on Jointly
Compressed Sensing in order to exploit the intra- and intersignal correlations present in the RSS measurements.

The authors in [19] also applied the theory of CS, this time in order to minimize the number of the APs needed for accurate position estimation. In another recent work, indoor localization was also approached via the CS framework [32]. The localization algorithm presented in [32] is based on the measurements transmitted from the APs, while it requires the MS to interact with a central unit that estimates its position. Under this scenario, CS theory is utilized in order to minimize the number of measurements exchanged with the central unit.

From a different perspective, authors in [33] minimize the localization error by utilizing high dimensional fingerprints collected using a diverse set of parameters controlling the operational frequency and the transmission power at each possible location. Although their approach optimizes the accuracy of already existing techniques, an exhaustive training phase is required. Compared to that work, our proposed multi-channel localization system adopts a reduced effort calibration phase while performing accurate location estimation.

\section{Signature Maps Properties}

In this Section, we present illustrative examples that highlight two major issues that affect multi-channel fingerprint based positioning. First, we discuss the effect of channel (frequency) changes on the RSS measured values and the implication of this phenomenon on localization systems. Second, we describe the spatio-frequency correlations of the RSS measurements and we provide motivation for utilizing these correlations to reduce the time and effort of the training procedure. Based on that, we introduce the Matrix Completion framework as a formal principal method for the recovery of low rank signature maps.

\subsection{Multichannel RSS Model}

We consider a typical WLAN positioning scenario where a set of APs are connected and a user carries a MS equipped with a wireless network card. An AP that listens to a specific channel, collects the packets transmitted from the MS at that channel, and records the corresponding RSS values in order to produce a signature map. During the location estimation phase, runtime RSS measurements transmitted from the MS are collected and compared with the fingerprints obtained during the training phase.

To demonstrate the impact of channel selection on the received RSS fingerprints, Fig. 3 illustrates the mean RSS values over time (in $\mathrm{dBm}$ ) collected from channels 1, 5, 9 and 13 , as a function of distance from a specific AP. In this experiment, we recorded real RSS measurements in a corridor of a laboratory area during midnight in order to ensure a stable environment. Fig. 4 shows the floor map of the experimental area along with the locations of some of the APs.

The plot reveals some very interesting characteristics regarding the relationship between distance and channel (frequency). Overall, we observe that as the distance increases, the RSS values generally tend to decrease. This is expected since the power of the received signal is highly 


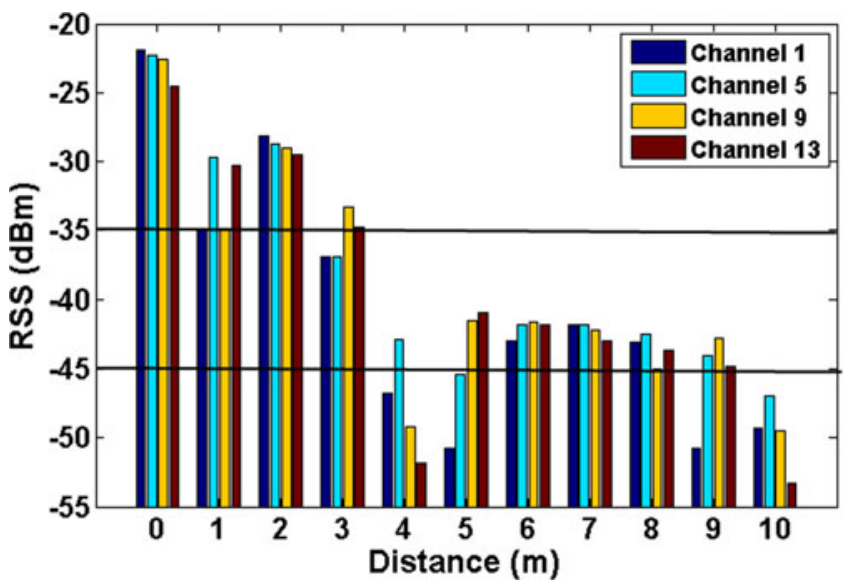

Fig. 3. An example of RSS value variation as a function of the distance from the AP. Each colorbar corresponds to the mean RSS measurements of a specific channel at a certain distance.

dependent on the distance. However, this decrease does not follow a smooth linear trend, e.g., observe that the mean RSS value in channel 1 is higher 2 meters away than it is 1 meter away from the AP. Furthermore, each channel is affected differently due to frequency related effects in radio propagation including multipath fading with propagation delays.

From a localization perspective, this plot reveals the sensitive relationship between location (distance) and channel (frequency) and that errors may result when this relationship is not taken into account. For example, an RSS measurement of $-35 \mathrm{dBm}$ may correspond to a 1 meter distance for channel 1 or to a 3 meter distance for channel 13. As a result, a fingerprint based localization scheme can produce an error of 2 meters when comparing a training fingerprint from, e.g., channel 1 with a runtime fingerprint from, e.g., channel 13. Similar effects can also be observed for a signal power equal to $-45 \mathrm{dBm}$ that corresponds to a distance of 5 meters for channel 3 or 9 meters for channel 13 .

This illustrative example suggests that a channel-unaware fingerprint comparison can lead to considerable location estimation errors. In order to provide accurate positioning, it is thus necessary to take into consideration the dynamic channel assignment during the training and the runtime phases

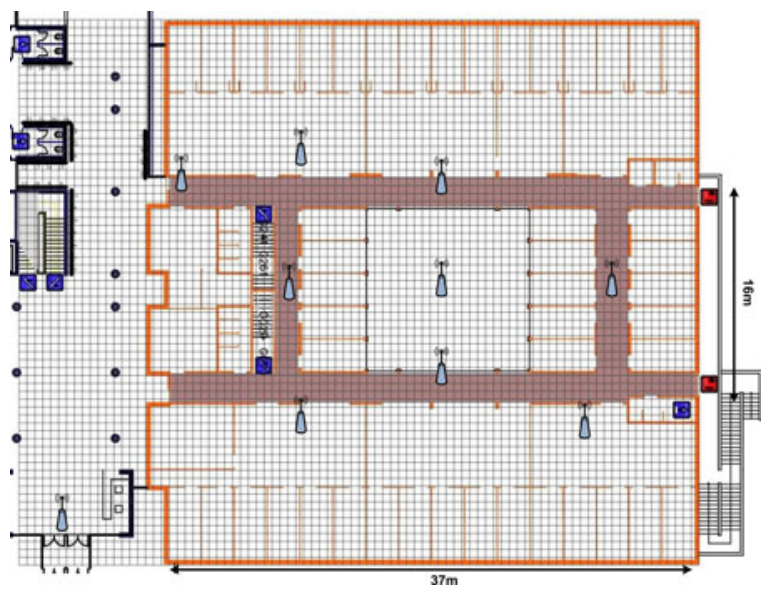

Fig. 4. Experimental set up at FORTH. The experiments were performed within the brown-colored area.

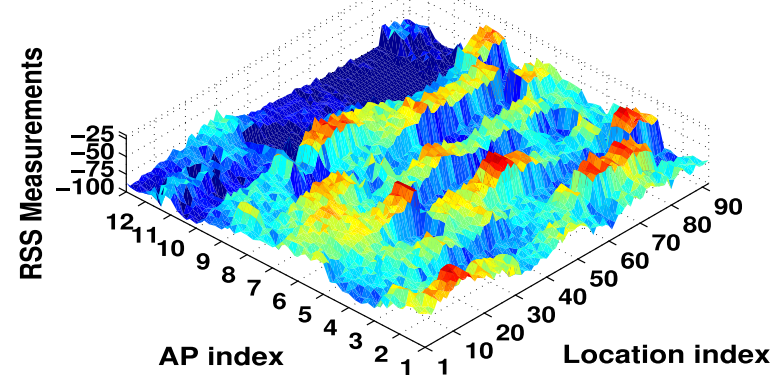

Fig. 5. Signature map of recorded RSS signatures from 12 APs for four channels at 91 different positions.

and to account for the different channels and the corresponding changes in RSS characteristics.

\subsection{RSS Correlation Model}

In this work, we argue that spatio-temporal correlations are prevalent in RSS measurements collected by a densely deployed AP infrastructure and that exploiting them can be extremely advantageous. To illustrate this behavior, we investigate the spatial and frequency correlations of RSS measurements collected from various APs at different channels and locations. More specifically, we consider the real RSS fingerprints encoded in a matrix, the signature map, shown in Fig. 5.

Each element of this matrix indicates the average RSS measurements from a specific AP, at a given channel and at a certain location. In this figure, we observe that (i) closely placed APs tend to produce similar RSS values and (ii) measurements from the same location but from different channels are also highly correlated due to the frequency invariant features of the RSS fingerprints.

The correlations of the measurements imply that the degrees of freedom of this matrix are much lower than its dimensions. The limited number of degrees of freedom results in a matrix that exhibits a rank much lower than its dimension. The low rank nature of the signature map can be observed in Fig. 6 which shows the normalized singular values of the signature map.

In the figure we observe that most of the energy is contained in the first few singular values, while noiselike phenomena result in additional singular values that exhibit much lower energy. The low rank nature of the signature map will play a pivotal role in our proposed localization scheme.

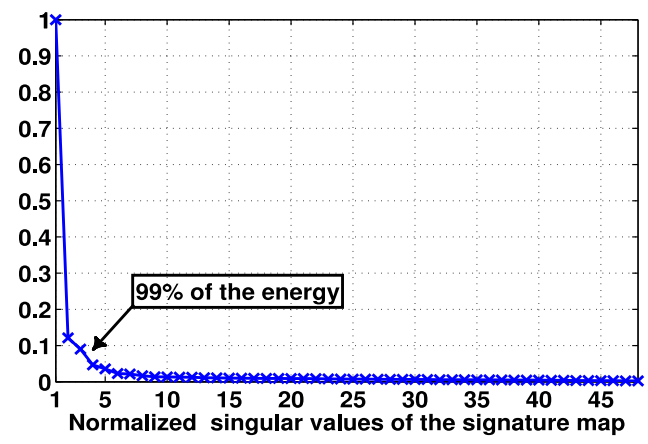

Fig. 6. Normalized singular values of the signature map. The rank of the signature map equals the number of non-zero singular values. 


\subsection{Recovery via Matrix Completion}

Matrix Completion is a recently proposed framework which considers the recovery of the complete set of matrix entries from a small number of randomly revealed entries [34]. Formally, let the matrix $\Psi \in \mathbb{R}^{K \times D}$ be the measurements matrix we wish to recover as precisely as possible. The goal according to the $\mathrm{MC}$ formulation is to recover the matrix $\Psi$ using a subset of $M$ entries, collected by the linear operator $\mathcal{A}: \mathbb{R}^{K \times D} \rightarrow \mathbb{R}^{M}$ such that

$$
y=\mathcal{A}(\Psi),
$$

where $y \in \mathbb{R}^{M}$ collects $M$ observed measurements.

In general, the recovery of the $K \times D$ entries of matrix $\Psi$ is impossible from $M \ll K \times D$ measurements since the linear system of equations described in (1) is underdetermined. The MC framework suggests that such a recovery is possible, provided that the rank of matrix $\Psi$ is small enough compared to its dimensions and that enough measurements are obtained. The rank of a matrix is defined as the number of linearly independent rows or columns and encodes critical information regarding the underlying data generation process.

To recover an estimate $\hat{\Psi}$ of the unknown matrix $\Psi$, the following rank minimization problem needs to be solved:

$$
\begin{array}{ll}
\text { minimize } & \operatorname{rank}(\hat{\Psi}) \\
\text { subject to } & \mathcal{A}(\hat{\Psi})=\mathcal{A}(\boldsymbol{\Psi})
\end{array}
$$

The low-rank matrix completion problem, as defined in (2) is NP-hard and thus cannot be solved efficiently. Fortunately, it was shown in [34] that the rank can be replaced by the surrogate nuclear norm provided that the linear map $\mathcal{A}$ satisfies a modified Restricted Isometry Property (RIP), i.e., it performs uniform random sampling in both rows and columns [35]. Consequently, the rank minimization problem can be approximated by the tightest convex problem

$$
\begin{array}{ll}
\text { minimize } & \|\hat{\boldsymbol{\Psi}}\|_{\star} \\
\text { subject to } & \mathcal{A}(\hat{\Psi})=\mathcal{A}(\boldsymbol{\Psi}),
\end{array}
$$

where the nuclear norm is defined as the sum of the singular values of $\boldsymbol{\Psi}$, i.e., $\|\boldsymbol{\Psi}\|_{\star}=\sum_{k=1}^{K}\left|\sigma_{k}\right|$.

It has been shown in [34] that the nuclear-norm minimization problem can recover the unknown low-rank matrix from $M \geq c D^{6 / 5} r \log (D)$ random measurements, where $D>K$ and $\operatorname{rank}(\boldsymbol{\Psi})=r$.

The problem in (3) can be reformulated as a semidefinite programming problem, in which case it can be solved by off-the-shelf solvers (e.g., CVX [36]). A limitation of this approach is that typically these algorithms converge slowly, especially when dealing with large matrices. Recently, efficient algorithms that take the characteristics of this specific optimization into account, have been proposed [37], [38]. Singular Value Thresholding (SVT) [38] is an iterative algorithm where during each iteration, a singular value decomposition step is applied first, followed by a projection onto the known elements. SVT relies on the fact that the unknown matrix is low-rank at each iteration and the auxiliary matrices that hold the observed measurements are sparse.

\section{Multi-Channel Based Localization}

In this Section, we describe our proposed multi-channel fingerprint based WLAN localization method. We consider a grid partition of the physical space into fixed structured cells. The proposed system is characterized by two phases; the training phase and the runtime phase.

\subsection{Training Phase}

Under the traditional fingerprint-based localization paradigm, during the training phase, RSS measurements have to be collected from the MS at each possible reference point, termed cell, and at each individual channel. In addition to the time and effort required in order to collect such a set of RSS measurements, physical constraints could make such a process unfeasible, since APs may not change channel during the collection of the training data. In order to minimize the duration of the calibration phase and overcome the limitations of traditional training, we propose to perform random sampling of the RF environment, where the APs collect RSS measurements from a randomly selected channel at each cell.

More specifically, during the calibration procedure of the training phase, each AP receives RSS measurements from a MS that moves to the cells that define the area of interest. These measurements are stored at the local signature map of each AP. The local signature map $\Psi_{j} \in \mathcal{R}^{C \times D}$ for the $j$ th $\mathrm{AP}$ is defined as

$$
\boldsymbol{\Psi}_{j}=\left(\begin{array}{cccc}
P_{1,1} & P_{1,2} & \cdots & P_{1, D} \\
P_{2,1} & P_{2,2} & \cdots & P_{2, D} \\
\vdots & \vdots & \ddots & \vdots \\
P_{C, 1} & P_{C, 2} & \cdots & P_{C, D}
\end{array}\right)_{C \times D}
$$

where $P_{c, i}$ corresponds to the time averaged RSS measurements received from channel $c$ at location $i$. $C$ represents the total number of channels and $D$ is the number of cells in the area of interest. Hence, the $c$ th row of $\boldsymbol{\Psi}_{j}$ is the vector of RSS measurements that the $j$ th AP receives at frequency $c$ from all $D$ reference points.

The LS receives $\Psi_{j}$ from each $\mathrm{AP}_{j} \in\{1, \ldots, J\}$, in order to create the signature map $\boldsymbol{\Psi}=\left[\boldsymbol{\Psi}_{1} ; \ldots ; \boldsymbol{\Psi}_{J}\right]_{C \cdot J \times D}$. The process of channel sub-sampling results in an incomplete signature map. The observed training signature map contains a subset $\Omega \subseteq[C J] \times[D]$ of $\Psi^{\prime}$ s entries, where $|\Omega|=\frac{C^{\prime}}{C}(C J \times D)$. Furthermore, the specific sampling pattern is defined by the sampling operator $\mathcal{A}$

$$
\left[\mathcal{A}_{\Omega}(M)\right]= \begin{cases}P_{c, i}, & (c, i) \in \Omega \\ 0, & \text { otherwise }\end{cases}
$$

The LS collects the incomplete matrix $\Psi$ (cf. Fig. 1 - Training phase) that satisfies the low-rank property required by the MC theory. Effective location sensing requires the recovery of the complete signature map $\hat{\Psi}$ that will be used during the runtime phase. Recovery of the unobserved RSS measurements can be achieved by solving the following optimization problem

$$
\min \left\{\|\hat{\mathbf{\Psi}}\|_{\star}: \quad\left\|\mathcal{A}_{\Omega}(\hat{\mathbf{\Psi}})-\mathcal{A}_{\Omega}(M)\right\|_{F}^{2}<\epsilon\right\},
$$


where $\hat{\Psi}$ is the recovered signature map, $\epsilon$ is the noise level, and $\|\cdot\|_{F}$ denotes the Frobenius norm. The optimization problem in (6) can be solved using the methods presented in Section 4.3. The computational complexity of the MC approach is $O(|\Omega|)$, as the dominant cost on recovering the signature map is proportional to the number of visiting cells and the number of sensed channels during calibration (i.e., the number of linear constraints imposed in (6)).

\subsection{Runtime Phase}

The new multi-channel localization scheme that we introduce in this work, considers the impact of frequency on the RSS propagation and utilizes this information during the estimation of the mobile user's location. A critical insight regarding the position of a MS is that the associated location vector is inherently sparse when considering a discretized physical space [28].

Particularly, we consider the sparse vector $\boldsymbol{b} \in \mathbb{R}^{D}$ where a non-zero component at the $i$ th position indicates the presence of the MS at cell $g_{i}$. For instance, the vector

$$
\boldsymbol{b}=[0,1,0, \ldots, 0]^{T},
$$

indicates that the MS is located at the second cell.

During the runtime phase, the MS broadcasts on all available channels and each AP, associated to a specific channel $k$, collects runtime RSS measurements from the MS at that channel. The localization process is performed at the LS where the runtime measurements $y_{j}$ from each AP are collected into the runtime fingerprint

$$
\boldsymbol{y}=\left[y_{1}^{\left(c_{1}\right)}, y_{2}^{\left(c_{2}\right)}, \ldots, y_{J}^{\left(c_{J}\right)}\right]_{J \times 1}^{T},
$$

where $J$ is the number of APs and $y_{j}^{\left(c_{j}\right)}=\frac{1}{N} \sum_{t=1}^{N} P_{j}^{\left(c_{j}\right)}(t)$ is the average value of RSS runtime measurements over time from $\mathrm{AP}_{j}$ at channel $c_{j}$. The channel information is acquired from the packet's channel information element transmitted from the mobile device.

Contrary to currently employed localization methods, the proposed multi-channel localization scheme considers the channel information of the runtime measurements. Formally, the concept of AP channel selection can be expressed in the following form:

$$
y_{j}^{\left(c_{j}\right)}=\overbrace{\left[\begin{array}{lll}
0 & 1 \ldots & \ldots
\end{array}\right]}^{\phi_{j}} \cdot \overbrace{\left(\begin{array}{cccc}
P_{1,1} & P_{1,2} & \cdots & P_{1, D} \\
P_{2,1} & P_{2,2} & \cdots & P_{2, D} \\
\vdots & \vdots & \ddots & \vdots \\
P_{C, 1} & P_{C, 2} & \cdots & P_{C, D}
\end{array}\right)}^{\Psi_{C \times D}} \cdot \overbrace{\left(\begin{array}{c}
b_{1} \\
b_{2} \\
\vdots \\
b_{D}
\end{array}\right)}^{b} .
$$

The unit vector $\phi_{j}$ has all elements equal to zero except $\phi\left(c_{j}\right)=1, c_{j}$ being the index of the channel where $\mathrm{AP}_{j}$ received runtime measurements.

Exploiting the sparsity in the spatial domain, efficient location sensing translates to the accurate detection of the non-zero coefficient of the sparse vector $b$. The signal ensemble can be expressed as:

$$
y=\Phi \Psi b \stackrel{\Theta=\Phi \Psi}{=} \Theta b+\epsilon,
$$

where $\boldsymbol{\Phi}=\left[\phi_{1}, \ldots, \phi_{J}\right], \Theta$ is a submatrix of $\boldsymbol{\Psi}$ that collects training fingerprints from the APs of the runtime frequency, and $\epsilon$ is the noise vector. The matrix $\Theta$ is a structured lowrank dictionary as it obeys the property $\operatorname{rank}(\Theta)=$ $\operatorname{rank}(\boldsymbol{\Phi} \boldsymbol{\Psi}) \leq \min (\operatorname{rank}(\boldsymbol{\Phi}), \operatorname{rank}(\boldsymbol{\Psi}))$, where $\boldsymbol{\Phi}$ is a full-rank matrix and $\Psi$ is low-rank (cf. Section 4).

Exploiting the low-rank and sparsity properties of the model in (10), we employ at the LS a sparse Bayesian learning with relevance vector machine (RVM) approach to localize the mobile user. The Bayesian framework associated with RVM, given a dictionary-dependent sparsity penalty, presents invariance properties leading to accurate sparse signal estimation, especially for structured dictionaries [39], [40]. Given the runtime measurements $y$ and a prior belief that $b$ is sparse in basis $\Psi$, the main objective is to formulate a posterior probability distribution for $b$. The adopted probabilistic framework introduces a prior over the sparse vector regularized by a set of hyperparameters $\alpha_{i}$ associated with each position in the area of interest that mitigate the prior. The hyperparameters individually control the strength of the prior over its associated weight.

Sparse Bayesian learning defines a zero-mean Gaussian prior with precision $a_{i}$ (inverse-variance) on each element of the sparse vector $b$

$$
p(\boldsymbol{b} \mid \boldsymbol{\alpha})=\prod_{i=1}^{D} \mathcal{N}\left(b_{i} \mid 0, a_{i}^{-1}\right) .
$$

The measurement vector $y$ is modeled using a Gaussian distribution with variance $\sigma_{0}^{2}$,

$$
p\left(\boldsymbol{y} \mid \boldsymbol{b}, a_{0}\right)=\left(2 \pi \sigma_{0}^{2}\right)^{-\frac{J}{2}} \exp \left(-\frac{\|\boldsymbol{y}-\boldsymbol{\Theta} \boldsymbol{b}\|^{2}}{2 \sigma_{0}^{2}}\right) .
$$

By employing Bayes' rule and the Gaussian likelihood model, the posterior probability for the sparse vector $b$ is defined as

$$
\begin{aligned}
p\left(\boldsymbol{b} \mid \boldsymbol{y}, \boldsymbol{a}, a_{0}\right) & =\frac{p\left(\boldsymbol{y} \mid \boldsymbol{b}, a_{0}\right) p(\boldsymbol{b} \mid \boldsymbol{a})}{p\left(\boldsymbol{y} \mid \boldsymbol{a}, a_{0}\right)} \\
& =(2 \pi)^{-\frac{D}{2}}|\boldsymbol{\Sigma}|^{-\frac{1}{2}} \exp \left(-\frac{1}{2}(\boldsymbol{b}-\boldsymbol{\mu})^{T} \boldsymbol{\Sigma}^{-1}(\boldsymbol{b}-\boldsymbol{\mu})\right),
\end{aligned}
$$

where $|\cdot|$ denotes the determinant of a matrix. The posterior mean $\mu$ and the covariance matrix $\Sigma$ are given by

$$
\boldsymbol{\Sigma}=\left(\boldsymbol{A}+a_{0} \boldsymbol{\Theta} \boldsymbol{\Theta}^{T}\right)^{-1}, \boldsymbol{\mu}=a_{0} \boldsymbol{\Sigma} \boldsymbol{\Theta}^{T} \boldsymbol{b}
$$

and $A=\operatorname{diag}\left(a_{1}, \ldots, a_{D}\right)$. The vector $a$ defines the hyperparameters over each component of the estimated sparse vector given the prior [41]. The diagonal elements of the covariance matrix provide confidence intervals (i.e., error bars) on the accuracy of the estimated components of the sparse vector $b$. Consequently, estimating the sparse vector $b$ translates in estimating the unknown variables $a, \mu$ and $\Sigma$.

The hyperparameters are estimated by marginalizing them over the sparse vector $b$. This is an iterative process where each iteration estimates $a$ and $a_{0}$ that maximize the marginal likelihood 


$$
\begin{aligned}
\mathcal{L}\left(\boldsymbol{a}, a_{0}\right) & =\log p\left(\boldsymbol{y} \mid \boldsymbol{a}, a_{0}\right) \\
& =-\frac{1}{2}\left[J \log 2 \pi+\log |\boldsymbol{C}|+\boldsymbol{y}^{T} \boldsymbol{C}^{-1} \boldsymbol{y}\right],
\end{aligned}
$$

with $C=\sigma_{0}^{2} I+\Theta A^{-1} \Theta^{T}$. After a number of iterations, a small fraction of $a_{i}$ remains relatively small indicating the non-zero components of the sparse vector $b$. Consequently, the estimated location of the mobile user is the cell $g$ that corresponds to the maximum component of $b$ (i.e., to the smallest $a_{i}$ )

$$
g=\arg \max \boldsymbol{b}=\arg \max p\left(\boldsymbol{y} \mid \boldsymbol{b}, \boldsymbol{a}, a_{0}\right) .
$$

\begin{tabular}{|c|c|}
\hline Alg & ithm 1. Localization via SBL \\
\hline Inpu & runtime measurements $y$. \\
\hline Out & at: estimated location $g$ \\
\hline 1) & Determine prior distribution per each cell (eq. (11)). \\
\hline 2) & $\begin{array}{l}\text { Estimate the posterior probability of the sparse vector } b \\
\text { from Bayes' rule (eq. (13)). }\end{array}$ \\
\hline & $\begin{array}{l}\text { a) Estimate the hyperparameters via maximizing } \\
\text { (eq. (15)). }\end{array}$ \\
\hline 3) & Estimate the location via (eq. (16)). \\
\hline
\end{tabular}

The runtime localization algorithm via sparse Bayesian learning is summarized in Algorithm (1).

\section{EXPERIMENTAL Results}

In this Section, we test and evaluate the proposed multichannel fingerprint based localization technique in an indoor WLAN environment. Real RSS data were collected in the corridors of the Institute of Computer Science (ICS) of the Foundation for Research and Technology Hellas (FORTH), an area of approximately $37 \times 16 \mathrm{~m}$. For this area, a grid-based structure was considered with a cell of size $1.2 \times 1.2 \mathrm{~m}$ (cf. Fig. 4). The experiment involved a total of 12 IEEE $802.11 \mathrm{~b} / \mathrm{g}$ APs $(J=12), 10$ of which were placed on the same floor and two were located on a different floor.

The AP operating system, Cisco IOS, is equipped with a special command that sets the device in scanner/monitor mode. When the AP is in monitor mode, it receives packets that contain the RSS information. In a real environment where the MS may not transmit enough packets per time unit, we trigger the device with the assistance of the infrastructure (i.e., we issue a ping command) to produce packet transmissions more frequently. Each AP uses the unique MAC address of the mobile device as a distinctive feature to recognize the packets of the transmitter that wants to be located.

During the training phase, the signature map was constructed as follows. RSS observations were collected for a period of 20 seconds for each of four channels $(C=4)$ of IEEE $802.11 \mathrm{~b} / \mathrm{g}$ (channels 1, 5, 9 and 13) over 92 cells $(D=92)$. The four partially overlapping channels where chosen in order to achieve higher spectrum utilization with minimum interference. The amount of calibration effort can be defined as

$$
\text { Time }=T_{s} \times C \times J \times D+T_{c s} \times C \times D+T_{w} \times D,
$$

where $T_{s}=20 \mathrm{~s}$ is the sensing period, $T_{c s}=140 \mathrm{~ms}$ is the time required for an AP to change communication channel,

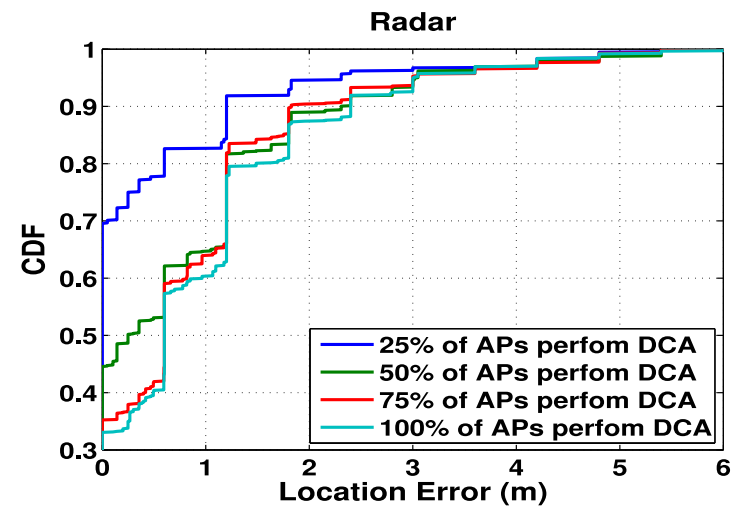

Fig. 7. CDF curves of the location error for the Radar system as a function of the number of APs that perform DCA, i.e., APs that may change the selected communications channel between training and runtime phase. The plot clearly indicates that changes of the RSS characteristics between training and testing have a profound effect on fingerprint matching and localization accuracy.

and $T_{w}=0.8 \mathrm{~s}$ defines the average time for a mobile user to change cell. Thus, in our setting, complete channel sensing requires $24.5 \mathrm{~h}$.

The systems are evaluated by their ability to identify the correct location, out of 46 distinct cells. The runtime dataset was collected on a typical weekday afternoon. We note here that the number of cells and online observations is comparable to those reported in [19] and [27].

The objectives of the experiments are twofold. First, we are interested in exploring and quantifying the effects that multiple channels have on the localization performance. This is a critical test, since localization algorithms typically assume a single communication channel. Second, we are aiming at identifying the benefits of using the proposed localization system, both in training and runtime phase. The accuracy of the reconstruction versus the number of measurements clearly describes the tradeoff between performance and time complexity during the training phase and localization accuracy during runtime.

\subsection{Effects of Dynamic Channel Assignment on State-of-the-Art Localization Techniques}

In this Section, we investigate the effects of frequency mismatch during training and runtime on the localization error of two state-of-the-art fingerprint based systems, the Radar [9] and the Horus [24]. In order to analyze the impact of dynamic channel assignment in localization accuracy, for this experiment only, we consider runtime measurements received in a stable environment, i.e., during midnight. We selected this setup since a stable environment minimizes the errors introduced by unexpected multipath and shadowing effects (i.e, from moving people and objects). We implemented the Radar positioning system by employing the nearest neighbor in signal space localization technique, while we implemented the Horus system by maximizing the posterior probabilities with respect to each possible position in the area of interest.

Figs. 7 and 8 show the CDF curves $(P|X| \leq x)$ of the location error as a function of the number of APs that adopt DCA for the Radar and the Horus systems, respectively. In each run we choose randomly the indexes of APs that 


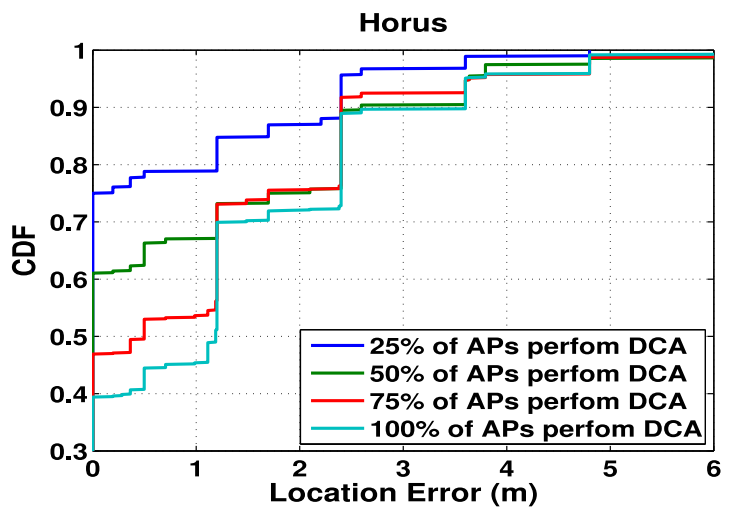

Fig. 8. CDF curves of the location error for the Horus system as a function of the number of APs that perform DCA. We observe that Horus exhibits a similar behavior to Radar with respect to the number of APS that may change channels between phases, demonstrating the additional localization error due to channel mismatch.

perform DCA while each curve in the graph is the average of 20 Monte-Carlo runs. The random channel assignment will lead to runtime RSS observations which are compared with RSS data observed at different frequencies and therefore different fingerprints.

We observe in the plots that as the number of APs that adopt DCA increases, the achieved localization accuracy decreases. Furthermore, frequency mismatch affects the location error for both systems, quite significantly. Particularly, in the case where only 25 percent of the APs perform DCA, the 90th percentile, (i.e., the value below which the 90 percent of the location error falls) is more than $1 \mathrm{~m}$, for both systems. Comparing the two systems, we observe that Horus is slightly more robust to the changes in channels compared to Radar.

In non-stationary indoor environments, each communication channel is affected differently due to various frequency related phenomena and multi-path effects. We analyzed the impact of adjacent and non-adjective channels on the location error for the two systems by performing two different experiments. During the training phase, RSS fingerprint signatures were collected on channel 1 that operates at center frequency $2.412 \mathrm{GHz}$ with bandwidth $5 \mathrm{MHz}$. In the first experiment, all the APs perform DCA to the adjacent partially overlapping communication channel 5 that operates at center frequency $2.432 \mathrm{GHz}$ with bandwidth $5 \mathrm{MHz}$ (i.e., the central frequency distance between the communication channels during the training and runtime phase is $20 \mathrm{MHz}$ ). In the second experiment all the APs perform DCA on the non-adjacent channel 9 that operates at center frequency $2.452 \mathrm{GHz}$ with bandwidth $5 \mathrm{MHz}$ resulting in central frequency distance of $40 \mathrm{MHz}$. Table 1 indicates the

TABLE 1

Location Error Statistics in Meters for Radar and Horus When the DCA Assigns to the APs Adjacent and Non-Adjacent Channels

\begin{tabular}{lccccc}
\hline & \multicolumn{2}{c}{ Radar } & & \multicolumn{2}{c}{ Horus } \\
\cline { 2 - 3 } \cline { 5 - 6 } & Mean & std & & Mean & std \\
\hline Adjacent Channels & 1.31 & 1.16 & & 1.24 & 1.53 \\
Non-adjacent Channels & 1.66 & 1.43 & & 1.51 & 1.61 \\
\hline
\end{tabular}

Observe that adjacent channel assignment results in lower mean location errors for both systems.

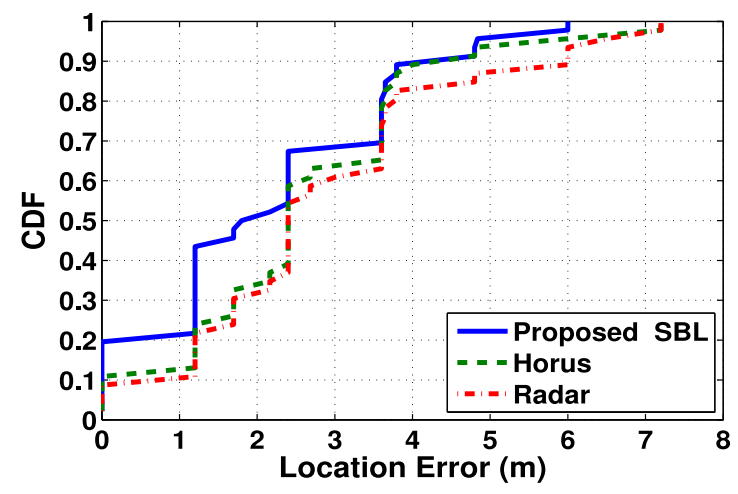

Fig. 9. The CDF of location error for the sparse Bayesian learning method, the Horus, and the Radar. During training, we perform full channel sampling.

location error statistics in meters for the two localization systems. We observe that adjacent communication channels result in higher localization accuracy for both systems. Specifically, adjacent channels are affected by invariant features leading in highly correlated RSS fingerprints varying in small $\mathrm{dBs}$ and thus resulting in small localization errors.

The above results highlight the need for considering the actual communication channels that are employed during both the training and the runtime phases. In the next Section, we compare the proposed channel aware-sparse Bayesian learning localization system with leading methods when all data is available.

\subsection{Localization via Sparse Bayesian Learning}

In the previous section, we presented experimental results on real data that reveal the influence of frequency mismatch on localization accuracy. In this section, we turn our attention to the evaluation of the proposed multi-channel localization method and compare its performance to the multi-frequency extension of the state-of-the-art Radar and Horus systems. We perform full frequency sensing during training while during runtime the correct channel information is considered via the matrix $\Phi$ as it was described in Section 5.2.

The CDF curves $(P|X| \leq x)$ in Fig. 9 illustrate the performance of the proposed sparse Bayesian learning localization method compared to channel-aware Radar and Horus. Observe that the 40 percent percentile (i.e., the number below which 40 percent of location errors falls) is $1.2 \mathrm{~m}$ for the proposed method, while for the Radar and Horus is $2.4 \mathrm{~m}$.

Fig. 10 illustrates the impact of the available infrastructure (i.e., number of APs) on the system's accuracy. The larger the number of APs, the higher the localization accuracy. We observe that the proposed technique achieves a lower mean location error compared to the other fingerprint-based systems. For instance, in the case of only three APs the mean location error of the proposed technique is $3.7 \mathrm{~m}$ while for the Horus and Radar is 5 and $5.7 \mathrm{~m}$, respectively. Interestingly enough, it appears that there is a critical number of APs required after which the accuracy of the system increases marginally ( 7 in our experiment).

In the aforementioned localization systems, the training fingerprints were collected from all channels at each location. However, as discussed in the previous sections, this 


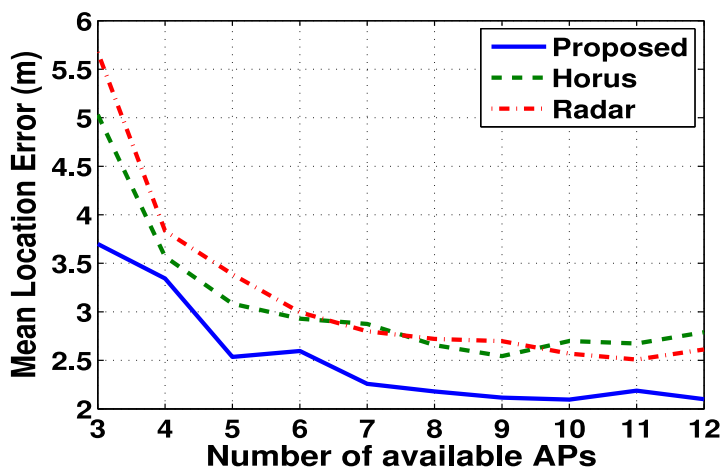

Fig. 10. Mean location error for various localization systems as a function of the number of available APs.

leads to a significant increase in labor and time that is required during the training phase. Next, we experimentally assert how spatio-frequency correlations can be exploited in order to significantly reduce the training time.

\subsection{Signature Map Estimation via Matrix Completion}

The performance gain in utilizing the appropriate channel during the localization process, motivates the need for an efficient estimation of the complete set of RSS fingerprints. The effectiveness of the proposed MC training technique is compared to the traditional Interpolation technique that serves as a baseline. The reconstruction problem presented in (6) is solved using the convex programming package CVX and the optimization approach SVT. ${ }^{1}$ We evaluated the performance of the proposed scheme with respect to both the reconstruction quality of the signature map and the corresponding localization error. The reconstruction error between the full signature map $\Psi$ and the estimated map $\hat{\Psi}$ is defined as

$$
R E=\|\hat{\Psi}-\boldsymbol{\Psi}\|_{F} /\|\boldsymbol{\Psi}\|_{F}
$$

First, the proposed MC-based RSS recovery technique is evaluated with respect to the number of sensed channels when training fingerprints are received from all 12 APs. Fig. 11 shows the recovery of the signature map as a function of the number of sensed channels. We observe that the performance of the proposed technique improves as the number of sensed channels increases. Furthermore, the proposed approach is able to achieve lower reconstruction error compared to the interpolation technique.

Fig. 12 illustrates the performance of our technique as a function of the total number of available APs, when the APs receive RSS measurements only from two channels (i.e., reducing calibration effort by half). Notice that the total number of available APs affects the structure of the signature map. Particularly, as the number of APs increases, more correlated RSS measurements are produced, thus the signature map exhibits a lower rank relative to its dimensions, which leads to a lower reconstruction error.

It is apparent that the MC-based approach provides lower reconstruction errors when compared to the Interpolation method for equivalent calibration effort. The proposed

1. For the implementation of MC and methods CVX and SVT we used the MATLAB codes included in the packages: http://cvxr.com/ $\mathrm{cvx} /$, http://svt.stanford.edu/index.html

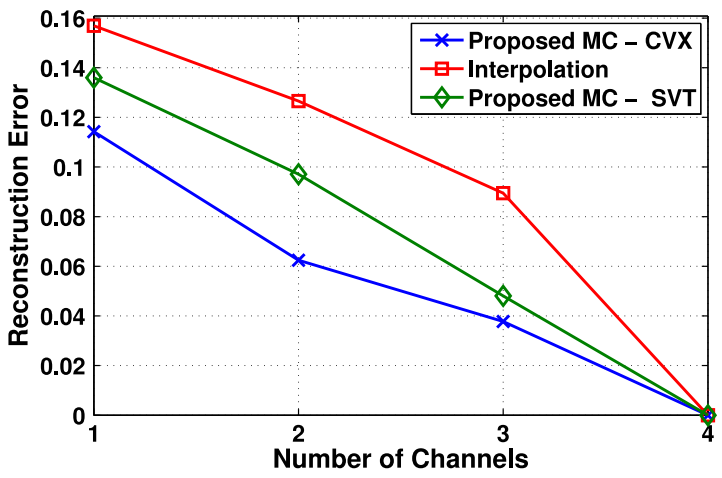

Fig. 11. The reconstruction error during the training phase as a function of the number of sensed channels. The experiment involves a total of 12 APs. We observe that MC with CVX achieves the lowest reconstruction error, half that of interpolation for the case of two sensed channels.

channel sub-sampling training approach can be adopted from various fingerprinting localization techniques in order to minimize the exhaustive calibration phase.

\subsection{Localization Performance with Recovered Signature Maps}

In this Section, we evaluate the effect of the proposed training technique during the runtime phase on various fingerprint based localization systems. Particularly, we are interested in determining how the errors in the reconstruction of the signature maps affect the localization performance.

Fig. 13 illustrates the CDF curves $(P|X| \leq x)$ of the proposed sparse Bayesian learning location method. As the number of sensed channels used during training increases, the performance of the proposed SBL technique improves. This behaviour is expected due to the fact that lower reconstruction errors during training result in lower localization errors during runtime. Particularly, we observe that the accuracy increases only slightly when sensing more than two channels. This behavior is consistent with the theoretical foundation of MC theory since the number of observed measurements required for accurate matrix recovery is indicated by the rank $(r)$ of the matrix. We note that sensing two channels (i.e., sensing 50 percent of the measurements in $12 \mathrm{~h}$ and $18 \mathrm{~min})$ corresponds to $M=2208(0.5 \cdot C J D, \mathrm{C}=4$, $\mathrm{J}=12, \mathrm{D}=92$ ) measurements which is above the minimum

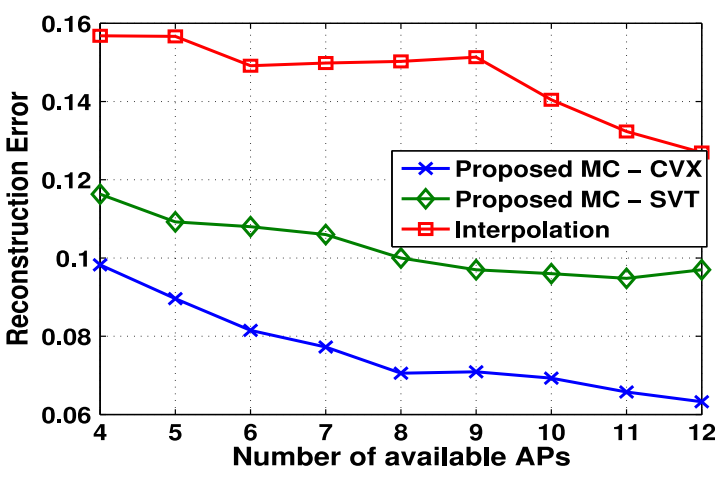

Fig. 12. Signature map reconstruction error during training phase as a function of the total number of available APs. In this experiment, the APs receive measurements only from 2 channels. The number of APs defines the structure of the signature map. 


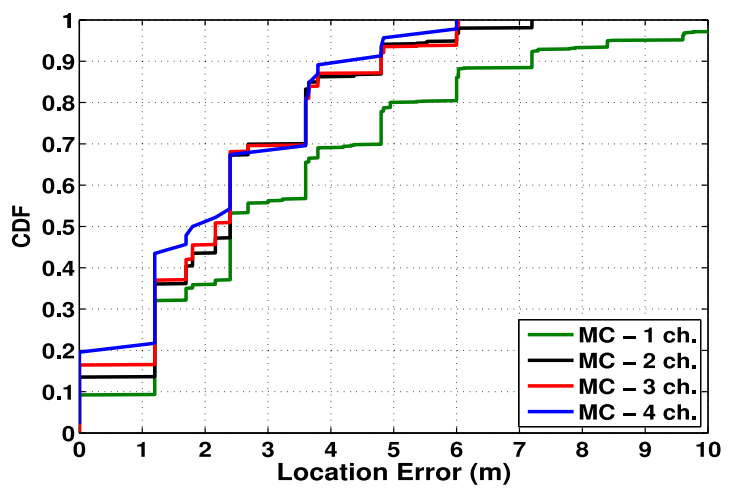

Fig. 13. The CDF curves of the location error for the proposed sparse Bayesian learning algorithm using the $\mathrm{MC}$-based technique as a function of the number of sensed channels during training.

bound of $c D^{6 / 5} r \log (D) \approx 1785$ measurements required by the theory of MC.

Fig. 14 illustrates the performance of the SBL, Horus, and Radar systems when one channel is sensed during the training phase. In Fig. 14a, all three systems adopt the MC-CVX strategy during training while in Fig. 14b the interpolation technique is used to construct the signature map.

It is clear that the proposed MC-based approach results in lower location errors when compared with the Interpolation method. Observe that the MC-based approach leads to improvements of the median location error (i.e., the values below which 50 percent of the location errors falls) in the order of 36 percent $(1.4 \mathrm{~m}), 21$ percent $(1 \mathrm{~m})$ and 25 percent

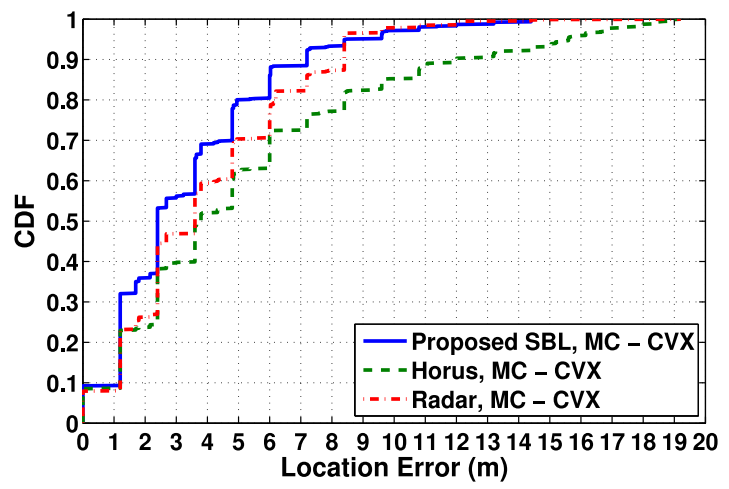

(a)

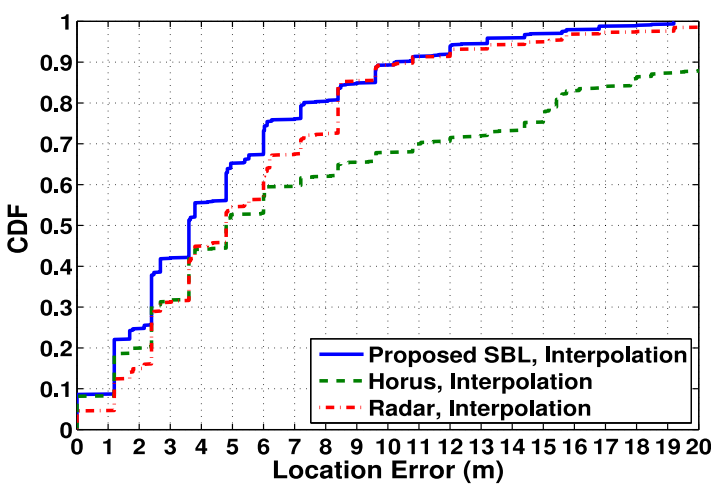

(b)

Fig. 14. The CDF curves of the location error for SBL, Horus, and Radar. One channel is sensed during training phase. (a) Location error using the proposed MC-based technique. (b) Location error using the Interpolation method.
TABLE 2

Average Location Error in Meters for the SBL, Horus, and Radar Systems and the MC and Interpolation Training Techniques as a Function of the Number of Sensed Channels during Training

\begin{tabular}{lcccccccc}
\hline & \multicolumn{3}{c}{ MC (Proposed) } & & \multicolumn{3}{c}{ Interpolation } & FS \\
\cline { 2 - 4 } \# Channels & $\mathbf{1}$ & $\mathbf{2}$ & $\mathbf{3}$ & & $\mathbf{1}$ & $\mathbf{2}$ & $\mathbf{3}$ & $\mathbf{4}$ \\
\hline Radar & 4 & 3.2 & 2.9 & & 5.7 & 3.4 & 3 & 2.8 \\
Horus & 5.2 & 3.5 & 3.1 & & 8.5 & 5.8 & 3.9 & 2.6 \\
SBL (Proposed) & 3.3 & 2.3 & 2.2 & & 4.8 & 3.2 & 2.8 & 2 \\
\hline
\end{tabular}

The FS column indicates the performance corresponding to full frequency sensing during training.

(1.2 m) for the SBL, the Horus, and Radar methods, respectively, over the Interpolation method. Moreover, the proposed MC-based approach results in lower maximum location errors.

Table 2 summarizes the mean location error of the three localization systems over different training techniques as a function of the number of sensed channels. As it can be seen, the proposed location sensing system outperforms the Radar and Horus systems in terms of location error. In contrast to other localization systems, the proposed SBL approach marginalizes the noise variance. Consequently, even in the presence of reconstruction noise introduced during training, the proposed localization algorithm results in lower mean location errors for both training techniques.

\subsection{Resources Utilization Analysis}

Table 3 summarizes the time calibration effort for performing random subsampling, as suggested in our approach, as a function of the number of sensed channels. The collected training RSS fingerprints are given to estimate the full signature map. The number of received fingerprints (third row) is a function of the number of sensed channels, the number of APs, and the dimensions of the space. Table 3 also provides the achieved estimation accuracy for the proposed MC and the interpolation methods. Observe that the proposed MCapproach, for a calibration time of 6 hours and 9 minutes (i.e., for one sensed channel) leads to improvements of 26 percent over the reconstruction error achieved by the interpolation method.

Although the power consumption of packet transmission and reception is highly hardware depended, studies indicate that in general, power consumption follows a linear trend with respect to the number of transmitted packets. For example, in [42] the authors proposed a linear function relating

TABLE 3

Calibration Effort during Training Time as a Function of Sensed Channels

\begin{tabular}{lcccc}
\hline \# Channels & 1 & 2 & 3 & FS \\
\hline Calibration time & $6 \mathrm{~h} 9 \mathrm{~m}$ & $12 \mathrm{~h} 18 \mathrm{~m}$ & $18 \mathrm{~h} 26 \mathrm{~m}$ & $24 \mathrm{~h} 32 \mathrm{~m}$ \\
\# Fingerpr. & 1104 & 2208 & 3312 & 4416 \\
Int. error & 0.157 & 0.126 & 0.089 & 0 \\
MC error & 0.114 & 0.062 & 0.038 & 0 \\
\hline
\end{tabular}

The last rows indicate the reconstruction error of the proposed MC technique and the Interpolation method. The FS column indicates the time effort for full frequency sensing. 
the percentage of energy consumption as a function of transmission time, $x$, given by $y=21.24 x+2.68$. A similar model was also developed in [43] where the power consumption (in Joules) for the download of $x$ bytes of data corresponds to $0.007(x)+5.9$, plus some overhead. Under the linearity assumption, transmission of packets over one out of four channels will result in close to 75 percent reduction in power consumption due to Wi-Fi communications and will have a profound effect on the lifetime of the mobile device.

\section{Conclusions}

Indoor localization is an exciting research field, with numerous applications. RSS based fingerprint methods offer high accuracy with minimal hardware interventions at the expense of a time consuming calibration process. Furthermore, in dynamic environments, frequent re-calibration is deemed necessary in order to maintain high localization accuracy, while dynamic channel assignments can lead to significant performance loss. In this work, we address these issues by proposing a novel multi-channel fingerprint-based indoor localization technique.

In the proposed scheme, estimation of the complete multi-channel signature map can be achieved from a small number of measurements over space and frequency (channel) by leveraging the power of Matrix Completion. Furthermore, the Sparse Bayesian Learning paradigm is introduced for the precise localization during the runtime phase.

To validate the merits of the proposed scheme, an extensive set of experiments was carried our using real data and a comparative analysis with state-of-the-art localization scheme was performed. Experimental results suggest that the proposed MC-based training technique can reliably estimate the signature map from partial information while sparse Bayesian learning outperforms traditional localization systems.

\section{ACKNOWLEDGMENTS}

This work was funded by the CS-ORION (PIAP-GA-2009251605) and HYDROBIONETS (ICT-GA-2011-287613) grants within the FP7 European Community. Sofia Nikitaki is the corresponding author.

\section{REFERENCES}

[1] Y. Gu, A. Lo, and I. Niemegeers, "A survey of indoor positioning systems for wireless personal networks," IEEE Commun. Surveys Tuts., vol. 11, no. 2, pp. 13-32, Mar. 2009.

[2] H. Liu, H. Darabi, P. Banerjee, and J. Liu, "Survey of wireless indoor positioning techniques and systems," IEEE Trans. Syst. Man Cybern. C, Appl. Rev., vol. 37, no. 6, pp. 1067-1080, Nov. 2007.

[3] J. Biswas and M. Veloso, "Multi-sensor mobile robot localization for diverse environments," in Proc. Robot Soccer World Cup XVII Symp., pp. 468-479, 2013.

[4] S. Papadakis and A. Traganitis, "Wireless positioning using the signal strength difference on arrival," in Proc. IEEE Int. Conf. Mobile Adhoc Sensor Syst., San Francisco, CA, USA, 2010, pp. $674-681$.

[5] D. Niculescu and B. Nath, "Ad hoc positioning system (APS)," in Proc. IEEE Global Telecommun. Conf., San Antonio, TX, USA, 2001, pp. 2926-2931.

[6] Y. Chen, W. Trappe, and R. P. Martin, "Attack detection in wireless localization," in Proc. 26th IEEE Int. Conf. Comput. Commun., Anchorage, AK, USA, 2007, pp. 1964-1972.
[7] J. Yang and Y. Chen, "Indoor localization using improved RSSbased lateration methods," in Proc. IEEE Glob. Telecommun. Conf., Honolulu, Hawaii, 2009, pp. 1-6.

[8] K. Kaemarungsi and P. Krishnamurthy, "Modeling of indoor positioning systems based on location fingerprinting," in Proc. 23th IEEE Int. Conf. Comput. Commun., Hong Kong, 2004, pp. 1012-1022.

[9] P. Bahl and V. N. Padmanabhan, "Radar: An in-building RF-based user location and tracking system," in Proc. IEEE 19th Annu. Conf. Comput. Commun. Soc., 2000, pp. 775-784.

[10] Y. Ji, S. Biaz, S. Pandey, and P. Agrawal, "Ariadne: A dynamic indoor signal map construction and localization system," in Proc. Int. Conf. Mobile Syst., Appl., Services, 2006, pp. 151-164.

[11] S. Chieochan, E. Hossain, and J. Diamond, "Channel assignment schemes for infrastructure-based 802.11 WLANs: A survey," IEEE Commun. Surveys Tuts., vol. 12, no. 1, pp. 124-136, First Quarter 2010.

[12] M. Boulmalf, T. Aouam, and H. Harroud, "Dynamic channel assignment in IEEE 802.11g," in Proc. Int. Wireless Commun. Mobile Comput. Conf., 2008, pp. 864-868.

[13] I. F. Akyildiz, W.-Y. Lee, M. C. Vuran, and S. Mohanty, “Next generation/dynamic spectrum access/cognitive radio wireless networks: A survey," Comput. Netw., vol. 50, no. 13, pp. 2127-2159, 2006.

[14] A. Taok, N. Kandil, and S. Affes, "Neural networks for fingerprinting-based indoor localization using ultra-wideband," J. Commun., vol. 4, no. 5, pp. 267-275, 2009.

[15] S. Nikitaki, G. Tsagkatakis, and P. Tsakalides, "Efficient training for fingerprint based positioning using matrix completion," in Proc. IEEE Eur. Signal Process. Conf., 2012, pp. 195-199.

[16] A. Haeberlen, E. Flannery, A. M. Ladd, A. Rudys, D. S. Wallach, and L. E. Kavraki, "Practical robust localization over large-scale 802.11 wireless networks," in Proc. 10th Annu. Int. Conf. Mobile Comput. Netw., 2004, pp. 70-84.

[17] B. Li, J. Salter, A. G. Dempster, and C. Rizos, "Indoor positioning techniques based on wireless LAN," in Proc. IEEE Int. Conf. Wireless Broadband Ultra Wideband Commun., 2006, pp. 13-16.

[18] X. Chai and Q. Yang, "Reducing the calibration effort for probabilistic indoor location estimation," IEEE Trans. Mobile Comput., vol. 6, no. 6, pp. 649-662, Jun. 2007.

[19] C. Feng, W. Au, S. Valaee, and Z. Tan, "Received signal strength based indoor positioning using compressive sensing," IEEE Trans. Mobile Comput., vol. 11, no. 12, pp. 1983-1993, Dec. 2012.

[20] M. Atia, A. Noureldin, and M. Korenberg, "Dynamic onlinecalibrated radio maps for indoor positioning in wireless local area networks," IEEE Trans. Mobile Comput., vol. 12, no. 9, pp. 17741787, Sep. 2013

[21] R. W. Ouyang, A. K. Wong, C.-T. Lea, and M. Chiang, "Indoor location estimation with reduced calibration exploiting unlabeled data via hybrid generative/discriminative learning," IEEE Trans. Mobile Comput., vol. 11, no. 11, pp. 1613-1626, Nov. 2012.

[22] A. Rai, K. K. Chintalapudi, V. N. Padmanabhan, and R. Sen, "Zee: Zero-effort crowdsourcing for indoor localization," in Proc. ACM 18th Annu. Int. Conf. Mobile Comput. Netw., 2012, pp. 293-304.

[23] T. King, S. Kopf, T. Haenselmann, C. Lubberger, and W. Effelsberg, "Compass: A probabilistic indoor positioning system based on 802.11 and digital compasses," in Proc. 1st Int. Workshop Wireless Netw. Testbeds, Exp. Eval. Charact., Los Angeles, CA, USA, 2006, pp. 34-40.

[24] M. Youssef and A. Agrawala, "The horus location determination system," Wireless Netw., vol. 14, pp. 357-374, Jun. 2008.

[25] A. M. Ladd, K. E. Bekris, A. P. Rudys, D. S. Wallach, and L. E. Kavraki, "On the feasibility of using wireless ethernet for indoor localization," IEEE Trans. Robot. Autom., vol. 20, no. 3, pp. 555559, Jun. 2004

[26] D. Madigan, E. Einahrawy, R. P. Martin, W.-H. Ju, P. Krishnan, and A. S. Krishnakumar, "Bayesian indoor positioning systems," in Proc. IEEE 24th. Int. Conf. Comput. Commun., Miami, FL, USA, 2005, pp. 1217-1227.

[27] H. S. Fang and T. Lin, "Principal component localization in indoor WLAN environments," IEEE Trans. Mobile Comput., vol. 11, no. 1, pp. 100-110, Jan. 2012.

[28] S. Nikitaki and P. Tsakalides, "Localization in wireless networks via spatial sparsity," in Proc. Annu. Asilomar Conf. Signals, Syst., Comput., Pacific Grove, CA, USA, 2010, pp. 236-239.

[29] E. Candès, J. Romberg, and T. Tao, "Robust uncertainty principles: Exact signal reconstruction from highly incomplete frequency information," IEEE Trans. Inf. Theory, vol. 52, no. 2, pp. 489-509, Feb. 2006. 
[30] D. Donoho, "Compressed sensing," IEEE Trans. Inf. Theory, vol. 52, no. 4, pp. 1289-1306, Apr. 2006.

[31] S. Nikitaki and P. Tsakalides, "Localization in wireless networks based on jointly compressed sensing," presented at the Proc. IEEE Eur. Signal Process. Conf., Barcelona, Spain, 2011.

[32] G. Tzagkarakis, A. Papakonstantinou, M. Papadopouli, P. Tsakalides, and D. Milioris, "Low-dimensional signal-strength fingerprint-based positioning in wireless lans," Ad Hoc Netw., vol. 12, no. 2, pp. 100-114, 2014.

[33] X. Zheng, H. Liu, J. Yang, Y. Chen, J. Francisco, R. P. Martin, and $\mathrm{X} . \mathrm{Li}$, "Characterizing the impact of multi-frequency and multipower on localization accuracy," in Proc. IEEE 7th Int. Conf. Mobile Adhoc Sens. Syst., 2010, pp. 156-165.

[34] E. Candès and B. Recht, "Exact matrix completion via convex optimization," ACM Commun., vol. 55, no. 6, pp. 111-119, 2012.

[35] B. Recht, M. Fazel, and P. Parrilo, "Guaranteed minimum-rank solutions of linear matrix equations via nuclear norm minimization," SIAM Rev., vol. 52, no. 3, pp. 471-501, Aug. 2010.

[36] M. Grant and S. Boyd, "CVX: Matlab software for disciplined convex programming, version 1.21," CVX Res. Inc., Austin, TX USA, 2011.

[37] R. H. Keshavan, A. Montanari, and S. Oh, "Matrix completion from a few entries," IEEE Trans. Inf. Theory, vol. 56, no. 6, pp. 2980-2998, Jun. 2010.

[38] J. Cai, E. Candès, and Z. Shen, "A singular value thresholding algorithm for matrix completion," SIAM J. Optim., vol. 20, no. 4, pp. 1956-1982, Jan. 2010.

[39] M. E. Tipping and A. Smola, "Sparse Bayesian learning and the relevance vector machine," J. Mach. Learn. Res., vol. 1, pp. 211-244, 2001.

[40] D. P. Wipf, "Sparse estimation with structured dictionaries," in Proc. Adv. Neural Inf. Process. Syst., 2011, vol. 24, pp. 2016-2024.

[41] M. E. Tipping and A. Faul, "Fast marginal likelihood maximisation for sparse Bayesian models," in Proc. 9th Int. Workshop Artif. Intell. Stat., Key West, FL, USA, 2003, pp. 3-6.

[42] G. Kalic, I. Bojic, and M. Kusek, "Energy consumption in android phones when using wireless communication technologies," in Proc. 35th MIPRO Int. Conv., 2012, pp. 754-759.

[43] N. Balasubramanian, A. Balasubramanian, and A. Venkataramani, "Energy consumption in mobile phones: A measurement study and implications for network applications," in Proc. ACM SIGCOMM Conf. Internet Meas., 2009, pp. 280-293.

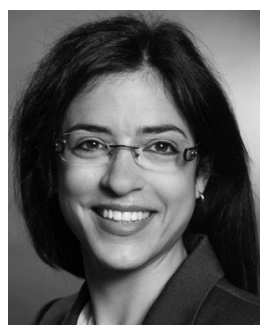

Sofia Nikitaki received the BS degree in informatics and telecommunications from the National and Kapodistrian University of Athens in 2007, and the $\mathrm{PhD}$ degree in computer science from the University of Crete in 2014. She is currently a research scientist at NEC Laboratories Europe, Germany. Her research interests lie in the area of signal processing with emphasis in sparse and low-rank models and applications in wireless networks.

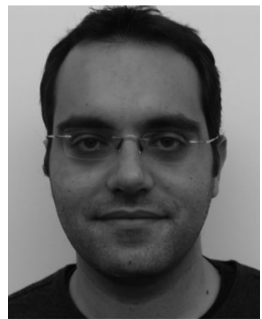

Grigorios Tsagkatakis received the BS and MS degrees in electronics and computer engineering from the Technical University of Crete (TUC), Greece, in 2005 and 2007, respectively. He was awarded the PhD degree in imaging science from the Center for Imaging Science at the Rochester Institute of Technology (RIT), in 2011. He is currently a postdoctoral fellow at the Institute of Computer Science-FORTH, Greece. His research interests include signal and image processing with applications in sensor networks and imaging systems.

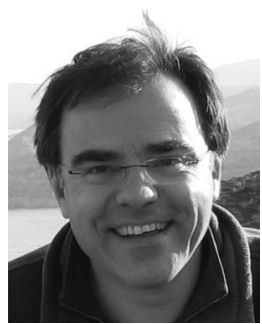

Panagiotis Tsakalides received the diploma in electrical engineering from the Aristotle University of Thessaloniki, Greece, in 1990, and the $\mathrm{PhD}$ degree in electrical engineering from the University of Southern California, Los Angeles, in 1995. He is a professor and the chairman of the Computer Science Department at the University of Crete, and the head of the Signal Processing Laboratory at the Institute of Computer Science (FORTH-ICS). His research interests lie in the field of statistical signal processing with emphasis in non-Gaussian estimation and detection theory, sparse representations, and applications in sensor networks, audio, imaging, and multimedia systems. He has coauthored more than 150 technical publications in these areas, including 30 journal papers. He has an extended experience of transferring research and interacting with the industry. During the last 10 years, he has been the project coordinator in six European Commission and nine Greek National Projects totaling more than $4 \mathrm{M€}$ in actual funding for the University of Crete and FORTH-ICS.

$\triangleright$ For more information on this or any other computing topic, please visit our Digital Library at www.computer.org/publications/dlib. 